\title{
Development of GPRS Communication System based on S3C2410A
}

\author{
Qunying Chen, Yunfang Hao \\ ZTE Telecommunication College, Xi'an Peihua Universty, Xi'an, 710125, China \\ 1179692610@qq.com,1605510498@qq.com
}

Keywords: S3C2410A; SIM300D; GPRS; development; serial ports communication; AT command; realViewMDK; secureCRT

\begin{abstract}
The GPRS communication system discussed in this paper which uses S3C2410A embedded microprocessor as main unit and SIM300D as the GPRS communication module. The development process of the system is introduced detail in this paper. It includes the construct of the development environment, hardware system connection and application programming. The system by Using the AT command can send, receive, read, delete text message, etc.
\end{abstract}

\section{Introduction}

Embedded system is a special combination of computer hardware and software. The characteristics of embedded system are "embedability", "Specificity", "computer system", namely, Each set of embedded system have its special applications and particular function. The embedded system consists of two parts: hardware and software. The core of the hardware part is embedded processor, configuration memory, I/O equipment, communication module, etc.; as for the software part, Software development platform is the center, above is the application programming interface API, below is the specific hardware characteristics of the board level support package BSP. In Embedded application system, software and hardware close cooperation and coordination to complete the system scheduled function.

GPRS is the General Packet Radio Service for short, it is a kind of mobile data service available to users of GSM mobile phones. It is often described as "2.5G", that is to say the mobile communication technology among the second generation $(2 \mathrm{G})$ and third generation $(3 \mathrm{G})$. Though GPRS is a transition technology as the existing GSM network to the third generation mobile communication evolution, but in many respects it has significant advantages, the development of GPRS technology is also very "economy", as long as the use of existing GSM network to GPRS development can be used widely, including through the mobile phone to send and receive e-mail, Browsing on the Internet, etc. System to complete the short message sending and receiving, must have a short message send and receive module, the GPRS module used in this system is SIM300D.

AT commands are commonly used in the connection and communication between the terminal device and PC. The European Telecommunications Standards (ETS) is defined in this way: At command is a set of common command that provides network services and applications through the terminal adapter (TA) from terminal equipment (TE) control mobile devices (ME).

AT commands just a set of command that Specified in the specific meaning, used to indicate the node, does not complete any operation by itself, must be analysis the significance of the AT command line, and then complete the corresponding operation according to the instruction in order to control the mobile terminal.

A system consists of a TE (such as a computer), a ME and their interface TA (such as a 3G data card). In practical application, usually the TA and ME are integrated together, that is general mobile devices, such as mobile phones. In this paper, the AT commands module in TA for processing the data interaction between ME and TE. 


\section{System Design}

2.1 Establishment of the Develop Environment. The development of embedded system, it is first necessary to establish a suitable cross development environment, there are many companies in the world provide different types of ARM development tools, according to different functions, there were compiled software, assembly software, Link software, debug software, Embedded operating system, function library, evaluation board, JTAG simulator, in circuit emulator, etc.. Some of the tools are provided a complete set, some tools are need to be used in combination. This system selects the RealViewMDK integrated development environment of Keil company as development tool and ULINK2 simulator.

MDK (Microcontroller Development Kit) is an arm development tool of keil (an ARM company), that is used to develop embedded application system based on ARM series micro controller. MDK contains industrial standard C compiler, macro assembler, a debugger, a real-time kernel components ,support all equipment based on ARM, can help the Engineer in accordance with plans to complete the project.

ULINK is a kind of USB to JTAG interface simulator of keil, at present the latest version is 2.0. It supports many chip manufacturers series processors of 8051, ARM7, ARM9 and cortex m3, Infineon C16x, Infineon XC16x, Infineon XC8xx, STMicroelectronics $\mu$ PSD etc. Power supply from USB interface of PC. ULINK2 includes not only all the characteristics of the ULINK USB to JTAG adapter but also increased the wire serial debugging (SWD) support, return clock and real-time agent function and apply to the standard Windows USB driver.

2.2 Establishment of the Develop Environment. SAMSUNG's S3C2410A 16/32-bit RISC microprocessor is designed to provide hand-held devices and general applications with cost-effective, low-power, and high-performance micro-controller solution in small die size. To reduce total system cost, the S3C2410A includes the following components: separate 16KB Instruction and 16KB Data Cache, MMU to handle virtual memory management, LCD Controller (STN \& TFT),NAND Flash Boot Loader, System Manager (chip select logic and SDRAM Controller), 3-ch UART, 4-ch DMA, 4-ch Timers with PWM, I/O Ports, RTC, 8-ch 10-bit ADC and Touch Screen Interface, IIC-BUS Interface, IIS-BUS Interface, USB Host, USB Device, SD Host \& Multi-Media Card Interface, 2-ch SPI and PLL for clock generation.

The S3C2410A was developed using an ARM920T core, 0.18um CMOS standard cells and a memory complier. Its low-power, simple, elegant and fully static design is particularly suitable for cost- and power-sensitive applications. It adopts a new bus architecture called Advanced Microcontroller Bus Architecture (AMBA).

The S3C2410A UART (Universal Asynchronous Receiver and Transmitter) provides three independent asynchronous serial I/O (SIO) ports, each of which can operate in Interrupt-based or DMA-based mode. In other words, the UART can generate an interrupt or a DMA request to transfer data between CPU and the UART. Each UART contains a baud-rate generator, a transmitter, a receiver and a control unit. The baud-rate generator can be clocked by PCLK or UEXTCLK. The transmitter and the receiver contain 16-byte FIFO sand data shifters. Data is written to FIFO and then copied to the transmit shifter before being transmitted. The data is then shifted out by the transmit data pin $(\mathrm{TxDn})$. Meanwhile, received data is shifted from the receive data pin $(\mathrm{RxDn})$, and then copied to FIFO from the shifter.The S3C2410A UART includes programmable baud rates, infra-red (IR) transmit/receive, one or two stop bit insertion,5-bit, 6-bit, 7-bit or 8-bit data width and parity checking.

Designed for global market, SIM300D is tri-band GSM/GPRS engine that works on frequencies, GSM $900 \mathrm{MHz}$, DCS $1800 \mathrm{MHz}$ and PCS1900 MHz. SIM300D provides GPRS multi-slot class 10 /Class 8 capability and supports the GPRS coding schemes CS-1, CS-2, CS-3 and CS-4.

SIM300D provide RF antenna interface. And customer's antenna should be located in the custom's mainboard and connect to module's antenna pad through micro strip line or other type RF trace but the impendence must be controlled in $50 \Omega$. 
The SIM300D is integrated with the TCP/IP protocol, Extended TCP/IP AT commands are developed for customers to use the TCP/IP protocol easily, which is very useful for those data transfer applications. Two serial ports can help you easily develop your applications, but they can not work at the same time. Two audio channels include two microphones inputs and two speaker outputs. This can be easily configured by AT command. Figure 1 shows the overall circuit connection diagram, S3C2410A processor receive AT command or control information that use PC keyboard input into Secure CRT Terminal Emulator through UART0, and through the uart1 control GPRS module, make its work in the appropriate model, and complete the short message sending or receiving etc.

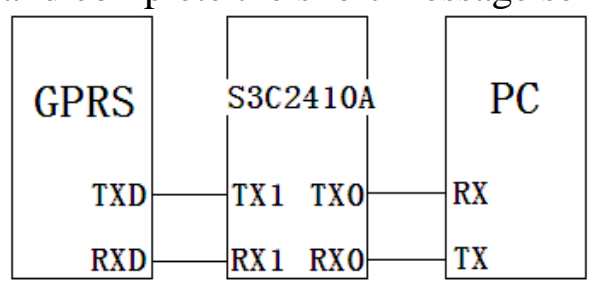

Figure 1. Circuit connection diagram of the system

In GPRS communication, according to different processing has different application, mainly have main program and function procedure. The main program completes system initialization and establishment of the GPRS module thread.

\section{Experimental Results}

Before Connect the computer serial port to S3C2410 UART0, connect well GPRS antenna, insert the SIM card(China Mobile card) into the cassette of GPRS module, connect the headset and microphone, running super terminal SecureCRT, select the correct serial port number, and set the serial port (115200 baud rate, parity check is None, 8 Data bits, 1 stop bit, no RTS/CTS). When GPRS module power on, GPRS auto reset, and enter the PC mode, in this mode, can directly control the GPRS module through the serial port of PC. Then, after GPRS module is initialized, by inputting different AT commands, can send, receive, delete Short Message, the experimental result is correct.

The AT commands related to receiving and sending short messages are as follows:

Table I. At commands related to receiving and sending short messages

\begin{tabular}{|c|c|}
\hline AT command & Function description \\
\hline AT & Connect with module \\
\hline $\mathrm{AT}+\mathrm{CMGF}=?$ & Returns the current working mode \\
\hline $\mathrm{AT}+\mathrm{CMGF}=\mathrm{n}$ & $\begin{array}{l}\text { Set the current mode of operation, } \mathrm{n} \\
=0: \text { PDU mode, } \mathrm{n}=1: \text { text mode }\end{array}$ \\
\hline $\mathrm{AT}+\mathrm{CMGS}$ & Send short message \\
\hline $\mathrm{AT}+\mathrm{CMGR}=\langle$ index $>$ & $\begin{array}{l}\text { Read the short message, where index } \\
\text { is the serial number of the message } \\
\text { in the current store }\end{array}$ \\
\hline $\mathrm{AT}+\mathrm{CMGL}$ & Output short message list \\
\hline $\mathrm{AT}+\mathrm{CMGD}=\langle$ index $>$ & $\begin{array}{l}\text { Delete short message, delete the } \\
\text { current store in the serial number of } \\
\text { index short message }\end{array}$ \\
\hline
\end{tabular}

3.1 Short Message Sending Process. In the super terminal window, enter the AT+CMGF=1, set the module working mode for the text mode.

After returning $\mathrm{OK}$, enter the $\mathrm{AT}+\mathrm{CMGS}=13 \mathrm{XXXX}-\mathrm{XXXXX}$ (enter the phone number), then press enter.

After ">"enter the message to be sent, Note that GPRS module does not support Chinese short message to be send now, can only send ASCII code.

3.2 Unread Short Message Receiving Process. In the super terminal window, enter the $\mathrm{AT}+\mathrm{CMGF}=1$, set the current working mode for the text mode.

Returns OK, then enter AT+CMGL, press enter. 
In the super terminal window, it can display the message that is not read in the short message list in the current store.

3.3 Read a Single Short Message Process. In the super terminal window, enter the AT+CMGF=1, set the current working mode for the text mode.

After returning $\mathrm{OK}$, enter the $\mathrm{AT}+\mathrm{CMGR}=$ index, the index is the number of destination messages that you want to read in the storage area, then press enter.

In the super terminal window, it can display the selected short message in the current storage area.

3.4 Short Message Deletion Process. Enter AT+CMGD=index in the super terminal window, index is the number of short messages to be deleted in the storage area and press enter.

Display OK in the super terminal window, showing that the deletion is complete.

\section{Conclusions}

This paper introduces the realization of GPRS communication system based on s3c2410A, described development process in detail. This system combine Wireless communication technology and Embedded technology together, make user terminal in a low cost, fast and reliable way into the GPRS network, data information can be simple, real-time transmission, provides a reliable guarantee for the user terminal safe and stable operation.

\section{Acknowledgment}

I would like to take this opportunity to thank Shenzhen Embest Technology Co., Ltd, This design is based on the EMBEST EDUKIT-IV experimental platform. Also thanks to my tutor----Yunfang Hao. In the process of composing this paper, she gives me many academic and constructive advices, and helps me to correct my paper. Except these, she also gave me the opportunity to do my teaching practice. At the same time, I would like to appreciate my literature teacher, who gives me useful literature knowledge and information in this paper. He is Duren Liu. At last, I am very grateful of my dear friends, Shipei Chen, Yaning Yan, who offered me the confidence and discuss with me about my paper.

\section{References}

[1] Samsung Electronics',Inc. S3C2410A microprocessor user's manual.2004.

[2] SIMCOM Ltd,SIM300D_HD_V2. 2006.

[3] Sony Ericsson Mobile Communications International. AT Commands Manual (Only for Reference). 2003.

[4] Shenzhen EMBEST Technology Co. Ltd, Development experiment and practice of embedded MDK based on S3C2410.2008.

[5] Cui Zhi-ting.Design and Implementation of SMS Management System Based on AT Command.2013.

[6] Liu Zhen. Research on PC machine and mobile phone communication based on GPRS/GSM module.2011.

[7] He Shi-jie ,Zhang Chun,Wang Yan-hong.The Design of GPRS System Based on S3C2410A.2011.

[8] LONG Guang-li.Design of GPRS system based on embedded system.2008.

[9] Li Min,Chen Wen,Wang Bao-qin.Design of embedded GPRS wireless communication system.2009.

[10] Liu Wei-ping.Research and Development of Communication between PC and Mobile device on Embedded system using GPRS.2011.

[11] Wang Rong-hui, Liu Gang. The Application of Embedded Sstemy in GPRS Based on the Chip of SC3 2410.2010.

[12] Wang Ru-tao, Huang Xing, Zhang Jin-long, Ding Jian.ARM9 GPRS Wireless Communication System Applied in Oilfield.2011. 